\title{
EVALUATION OF INHOMOGENEITIES IN HISTOLOGICAL STRUCTURES (CARTILAGE, RETINA)
}

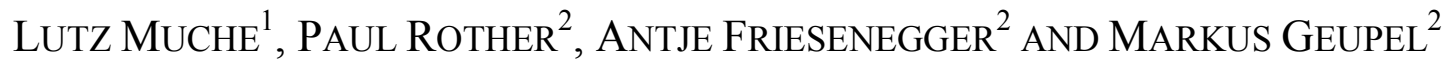 \\ ${ }^{1}$ Am Mühlteich 22, D-09599 Freiberg, Germany, ${ }^{2}$ Institut für Anatomie, Justus-von-Liebig-Straße 13, \\ Universitätsklinikum Leipzig, D-04103 Leipzig, Germany \\ (Accepted April 20, 2000)
}

\begin{abstract}
This paper investigates histological tissues by means of image analysis and spatial statistics. For the quantification of cell frequencies and accumulations two statistical characteristics, intensity function and cluster density, are suggested. The samples are histological sections of human articular cartilage and human retina considered in view of changes during the ageing process. The articular cartilage is characterized by continuous changes of both functions, the cell intensity as well as the clusterization. In contrast, the retina is a trilaminar structure formed in the early embryonic stage without changes by ageing.
\end{abstract}

Keywords: articular cartilage, clustering, intensity function, layer formation, retina

\section{INTRODUCTION}

Measurements at histological preparations lead to the phenomenon of inhomogeneity. The variation of interesting parameters shows both random fluctuations and systematic changes. The latter are well pronounced in cartilaginous tissue, exceptionally in cases, where it is covering as hyaline cartilage the articular surface without perichondrium. The systematic inhomogeneity of the articular cartilage has been evaluated quantitatively in detail. The extension of chondrocytes and their volume density has been studied in Löffler et al. (1993), as well as the orientation of the cells in Löffler et al. (1995). Additionally, the numerical intensity and the level of clustering of cells in an articular cartilage is characterized by a systematic change. In the direction from the articular surface to the bone the intensity is decreasing, whereas the clusterization is increasing (Schreitter, 1993). Based on samples of facies articularis navicularis of human caput tali, a systematic change of intensity and clusterization has been shown depending on the ageing process (Friesenegger, 1996).

Furthermore, a conspicuous feature of differentiation, described only qualitatively until now, is the phenomenon of layer formation, observable in cortex cerebri or in the human retina. Initially, there is one cell layer in the wall of ocular vesicle. Through the time a migration of a part of these cells causes to generate a second layer and again, a group of cells generates a third inner neuroblast layer. A quantitative evaluation of those layer formations with the aim of a better understanding of the prenatal and postnatal stage of development is desirable.

The questions are investigated by use of histological sections that means in planar, approximately twodimensional samples. Stereological corrections are not made. Earlier investigations have shown that, in this case, statements on ageing processes derived from histological sections in principle do not change during the addition of stereological corrections (Ranke and Ranke, 1989). An important fact of our consideration is a shrinkage of the tissue during the fixation around a half of its original thickness. But the connected decreasing of the cells is not relevant for our conclusions and is taken into account by the choice of the radius of neighbourhood for the estimation of the cluster density, respectively.

This paper presents a mathematical and computational approach for a numerical evaluation of those structures. The cells in a tissue are idealized by a point sample. The next two sections discuss the intensity function and the cluster density as tools to describe point samples and describe procedure to estimate these functions.

\section{PROCEDURE AND MATHEMATICAL BACKGROUND}

For the investigation the following haematoxylineosin-stained histological samples have been available: 
- 48 sections of articular cartilage (femoral head, talus head)

- 65 sections of human eyeballs of all age groups

- 10 sections of embryonic eyes of the first third of pregnancy.

The thickness of all sections is $10 \mu \mathrm{m}$, their orientation is perpendicular to the tissue surface. The $(x, y)$-coordinates are recorded of the centre of the cut parts of the nuclei. In the retina they are extracted with the image analysis system SIS automatically. In the articular cartilage sections this process was realized manually in first time, later also by help of image analysis using a measurement function, because an automatical detection of the coordinate of these nuclei is not possible. All coordinates are given in $\mu \mathrm{m}$; they describe the true locations of the cell nuclei in the histological samples.

This chapter gives a brief description of the procedure used to estimate intensity functions and cluster densities. The sample is given in form of a random point set in a bounded planar rectangle window $W=[0, \mathrm{a}] \times[0, \mathrm{~b}]$. Each point represents a cell. Its locations are given in Cartesian coordinates $\left(x_{i}, y_{i}\right), i=1,2, \ldots, n$ and $0 \leq x_{i} \leq a$ and $0 \leq y_{i} \leq a$. The coordinate system is chosen so that the $x$-axis represents the surface of a tissue, whereas the $y$-axis leads into the depth of this structure.

The mean number of points per unit area is called the intensity $\lambda$ of the sample. $\lambda$ is inversely proportional to the mean size of cells. (Clearly, the number of cells and therefore the intensity increases with the sectional thickness. But the preparations investigated here all have the same thickness of 10 $\mu \mathrm{m}$. Therefore the dependence on sectional thickness is not considered during this paper.) For a stationary (homogeneous) sample, $\lambda$ would be constant in each region of $W$. Additionally, for a stationary point pattern $\lambda$ is constant in each window, independently of its choice. For general definition and further properties see Stoyan et al. (1995), for example. Characterizing the non-stationarity of the sample, the intensity is assumed to be variable in the $y$-coordinate, since the structure is a gradient structure in the sense of Hahn et al. (1999). That means, the intensity function $\lambda(y)$ is defined to be the expected number of points in a differential small stripe $[0, a] \times[y, y+d y]$, divided by the area of the stripe $a . d y$.

The intensity function is estimated by use of a kernel function $h(\delta)$, given by

$$
h(\delta)= \begin{cases}\frac{3}{4 e_{\lambda}\left(1-\frac{\delta^{2}}{e_{\lambda^{2}}}\right)} & \delta^{2}<e_{\lambda^{2}} \\ 0, & \text { otherwise }\end{cases}
$$

the so-called Epanečnikov- kernel, cf. Hahn et al. (1999). Here $e_{\lambda}$ is the band width. The estimator $\hat{\lambda}(y)$ of the intensity function is

$$
\hat{\lambda}(y)=\frac{1}{a} \sum_{i=1}^{n} h\left(y-y_{i}\right) .
$$

That means, those points being close to $y$ contribute to $\lambda(y)$, independently of its $x$-coordinate. Points outside of this neighbourhood are without influence.

For $y$-coordinates near to the boundary of $W$ in $y$ direction, $\mathrm{b}-\mathrm{y}<e_{\lambda}$ the considered neighbourhood is reached outside of $W$, where information about further points is not available. This lack is compensed by an edge correction as follows. For any $y$ of distance $\varepsilon=\min (\|\mathrm{y}\|,\|\mathrm{b}-\mathrm{y}\|)<e_{\lambda}$ the value $\lambda(y)$ is increased by a factor $q$ which is the ratio between the measure of the complete kernel and that part of kernel being inside of the window $W$

$$
q=\frac{\int_{-e_{\lambda}}^{e_{\lambda}} h(\delta) d \delta}{\int_{-e_{\lambda}}^{\varepsilon} h(\delta) d \delta}=\frac{4 e_{\lambda}}{2 e_{\lambda}+3 \varepsilon\left(1-\frac{\varepsilon^{2}}{3 e_{\lambda}^{2}}\right)}, \quad \varepsilon \geq 0
$$

A cluster is an accumulation of points in a bounded region characterized by a higher point density than the environment. Shape and expansion of the clusters can be very different. Some characteristic to compare and classify clusters can be their circumscribing radius or their number of members. But the affiliation of a point to a cluster is not defined uniquely, in general. Overlappings of neighbouring clusters are possible. The function introduced in the following is a measure characterizing location and richness of clusters. The cluster density $v_{\mathrm{r}}(y)$ is defined to be the mean number of points $\left(x_{i}, y_{i}\right)$ in a circle of radius $r$ around an arbitrary point $(x, y)$ without counting $(x, y)$ itself. This parameter $r$ is called radius of neighbourhood. The cluster density gives information about expansion and location of regions of higher density. In general, there will not be a point having exactly the coordinate $y_{i}=y$. Therefore the estimation of the cluster density is based on a weighted mean value on those points, being in a stripe $[0, a] \times\left[y-e_{v}, y+e_{v}\right]$. 
The weight $w_{i}$ of a point $\left(x_{i}, y_{i}\right)$ is determined by a kernel function $k(\delta)$, analogously to $h(\delta)$, where $e_{\lambda}$ is just replaced by $e_{v}$. As above, only the distance in $y$ direction is relevant; the weight is

$$
w_{i}=k\left(\left\|y-y_{i}\right\|\right) \text {. }
$$

Let $n_{i}$ be the number of neighbouring points located in a circle of radius $r$ centering in $\left(x_{i}, y_{i}\right)$ without counting $\left(x_{i}, y_{i}\right)$ itself. Then the estimator for the cluster density is given by

$$
\hat{v}_{r}(y)= \begin{cases}\frac{\sum_{i=1}^{n} n_{i} w_{i}}{\sum_{i=1}^{n} w_{i}}, & \text { if } \sum_{i=1}^{n} w_{i}>0 \\ 0, & \text { otherwise. }\end{cases}
$$

The cluster density represents the mean number of neighbours being closer than $r$ to a given point of reference without consideration of their distances and locations in particular.

\section{SAMPLES AND RESULTS}

In the following, the three samples shown in Figs. 1,2 and 4 , are considered and some estimates are shown in Figs. 3, 5 and 6.

The computational procedure investigates a given point sample and provides estimates of the intensity function $\lambda(y)$ and the cluster density $v_{r}(y)$ having the following properties:

- $\quad \lambda(y)$ and $v_{r}(y)$ are meaningful functions to show tendencies and quantify changes in the cell configurations. They indicate layer formations or cluster locations in tissues.

- $\quad \lambda(y)$ and $v_{r}(y)$ do not give information about the distribution of the point samples along the $x$-axis. Under the assumption that the sample is homogeneous in $x, \lambda(y)$ and $v_{r}(y)$ are independent of the choice of the breadth $a$ of the observation window $W$.

- The estimations $\hat{\lambda}(y)$ and $\hat{v}_{r}(y)$ are influenced by the choice of the half band widths $e_{\lambda}$ and $e_{v}$, respectively.

The choice of the half band widths $e_{\lambda}$ is very important for the quality of the estimated function. If the half band width is too small, the estimated curve will be rough and oscillating. On the other hand, if the half band width is too large, the estimated functions will be too much smooth, without great fluctuations. Both cases preclude the interpretation of the results. Between these extremum cases there is an optimum value for $e_{\lambda}$ and $e_{v}$, where the estimation is most meaningful. The problem of choice of this optimum value is studied in the literature intensively, (Silverman, 1986). An empirical starting value is $e_{\lambda}=0.05 \sqrt{ } 5 / \lambda=0.05 \sqrt{ } 5 \mathrm{ab} / \mathrm{n}$ ). Also the radius of neighbourhood $r$ is of importance. In some cases it can be useful to adapt $r$ inversely proportional to the value of $\lambda(y)$,

$$
r(y)=\frac{\lambda}{\lambda(y)} r .
$$

This choice is based on the assumption that the cluster extension is positively and the cell size is negatively correlated with the intensity function. With increasing point density the extensions of the clusters decrease as well as the cells within.

\section{CONCLUSIONS}

The introduced intensity function gives the possibility to determine the cell-intensity layer specifically and continuously from the tissue surface into the depth. This enables a better analysis of the changes of cell numbers during the ageing process, including a quantification of the depth of these changes or of the layers.

Our evaluations explain interesting correspondences and differences of the layer-specifical changes of the numerical cell density of articular cartilage in the ageing process. The intensity function shows that systematic changes during ageing of the layer structure in the human retina in the postnatal period do not exist. Consequently, the histology of the retina turns out to be ageing stable, like many other microscopical structures of the central nervous system. In contrast to that, the intensity of a trilaminar image of the cells follows uni- or bilaminar preliminary stages in the early embryonic period. The precise recording of the depth depending changes of the clusterization is meaningful for the normal and pathological histology. This fact is shown clearly by the curves in Figs. 3, 5, 6.

The investigations were aimed at quantification and graphic demonstration of systematic inhomogeneities. Nevertheless, in each sample there exist a statistical inhomogeneity. Therefore the choice of the half band width must be effected so that the systematical trend is 
good visible, whereas small random inhomogeneities in the curves do not completely disappear, see Figs. $6 \mathrm{a}, \mathrm{b}, \mathrm{c}$. The phenomenon of clusterization starts in a certain depth for a sufficient high intensity. Therefore the estimated cluster density based on the adapted radius of neighbourhood seems to be more meaningful than the cluster density for a constant radius, cf. Figs. $6 \mathrm{~b}$ and $6 \mathrm{c}$.

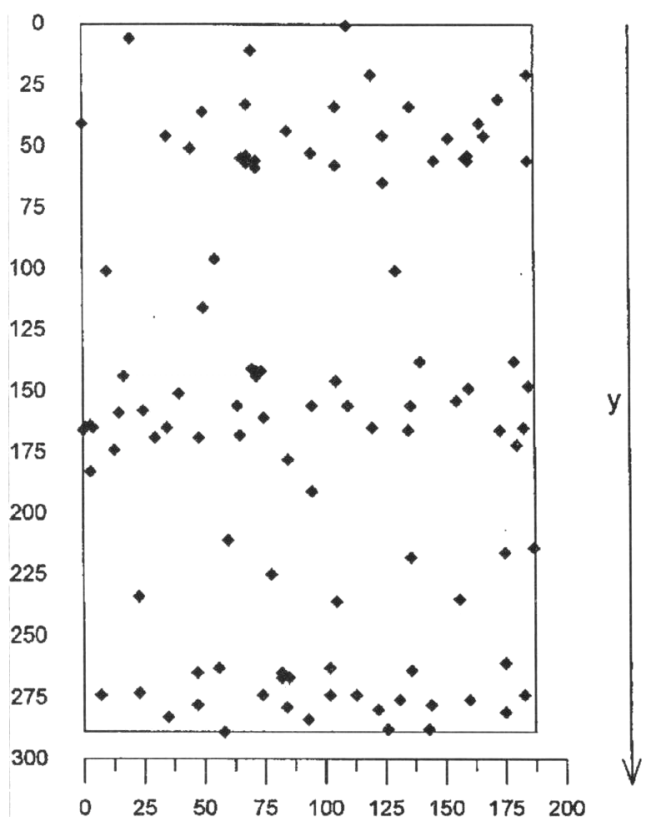

Fig. 1. Sample of human retina of a 64 years old patient, size $196.9 \mu \mathrm{m} \times 297.9 \mu \mathrm{m}, 105$ points. Different layer formations are visible.

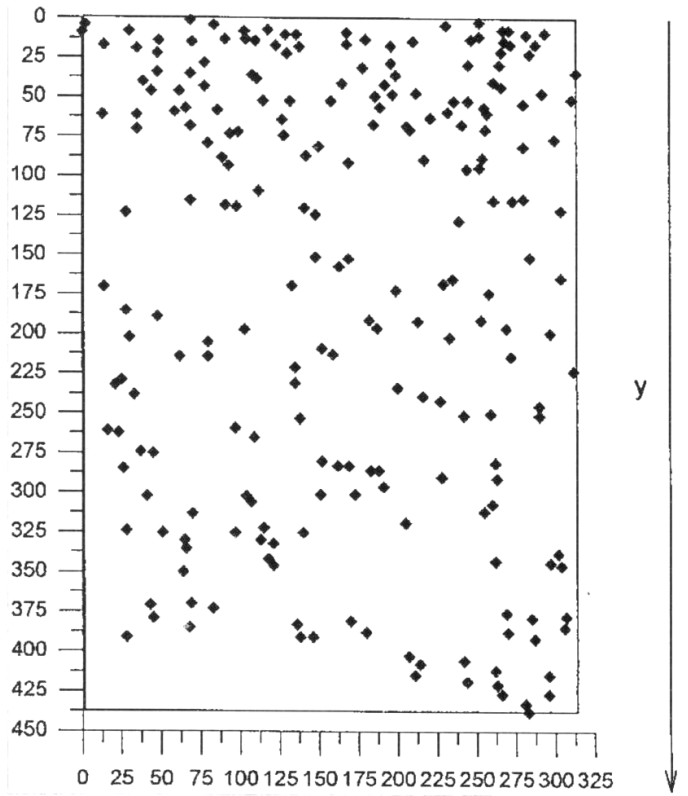

Fig. 3. Sample of articular cartilaginous tissue of a newborn child, size $153.0 \mu \mathrm{m} \times 363.0 \mu \mathrm{m}, 303$ points. The frequency of the points decreases continuously with increasing depth.
The procedure introduced here is recommendable for further fields of relevant functional and clinical histology. Systematic inhomogeneities are observed in the normal and by rheumatic diseases changed articular capsule. Number and distinction of ganglionic layers in the grey substance of the central nervous system are important functional parameters.

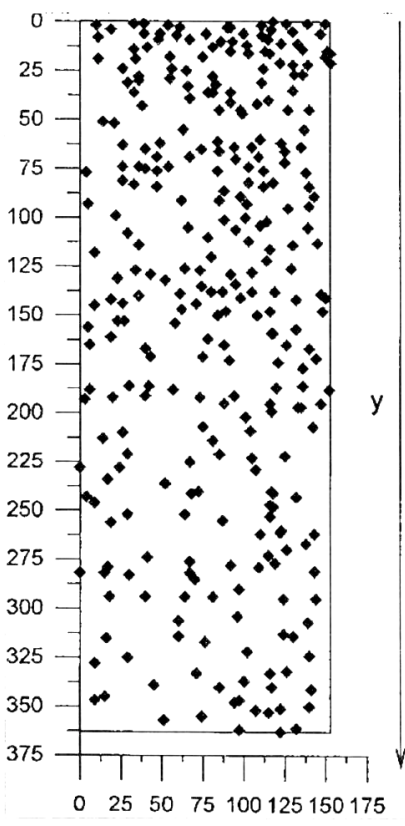

Fig. 2. The estimated intensity function for the sample given in Fig. 1. for a half band width of $e_{\lambda}=29.0 \mu \mathrm{m}$. The curve shows a clear subdivision into different layers.

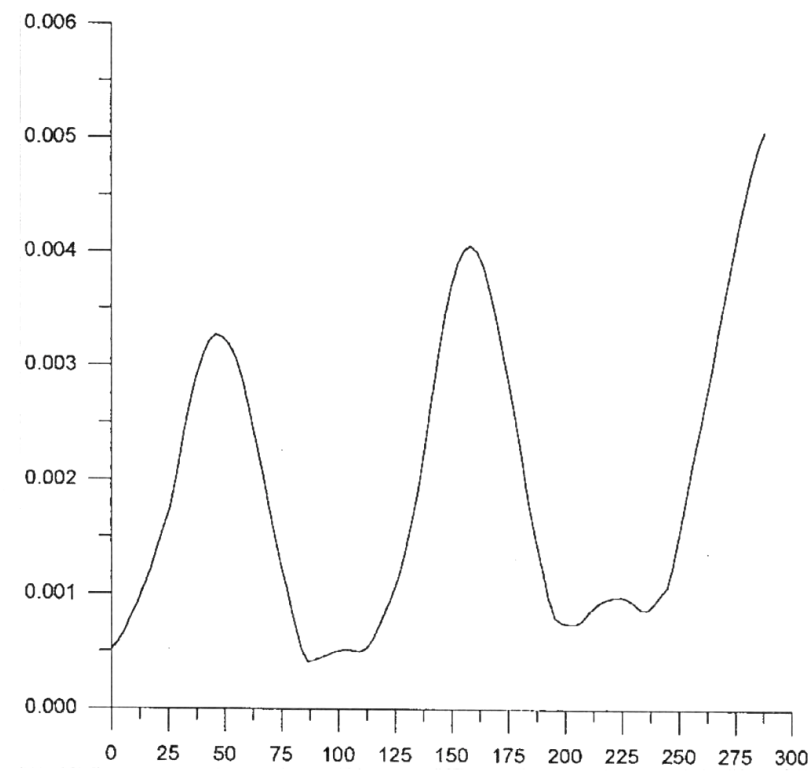

Fig. 4. The estimated intensity function for the sample given in Fig. 3. for a half band width $e_{\lambda}=41 \mu \mathrm{m}$. There is no clusterization at all. 


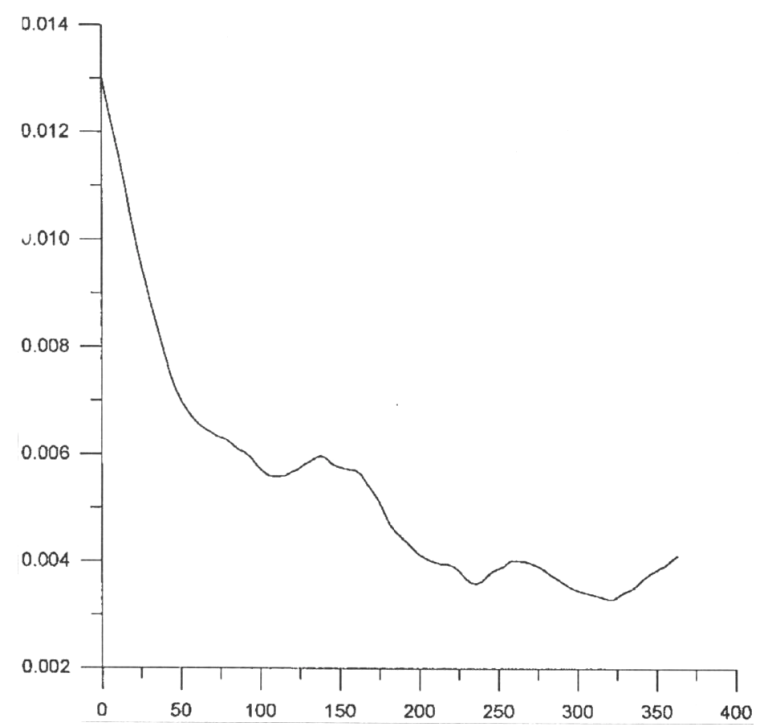

Fig. 5. Sample of articular cartilaginous tissue of a 57 years old patient, size $314.0 \mu \mathrm{m} \times 437.0 \mu \mathrm{m}, 226$ points. Additionally to the variable point frequency there are centres of clusterization.

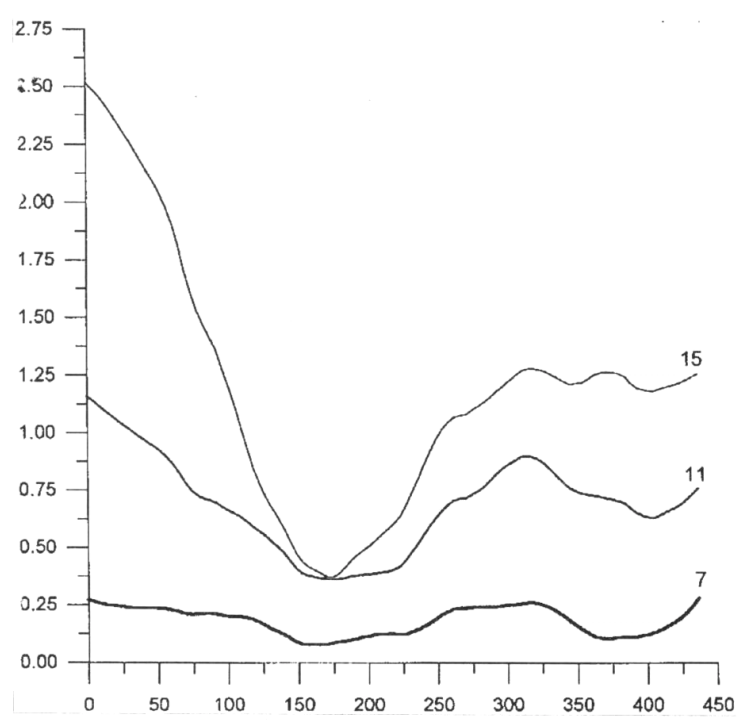

Fig. 6b. The cluster density for the sample given in Fig. 2 for a half band width $e_{v}=60 \mu \mathrm{m}$ for constant cluster radii of 7,11 and $15 \mu \mathrm{m}$.

\section{ACKNOWLEDGEMENT}

The authors are grateful to the anonymous referees for some critical comments and useful suggestions.

\section{REFERENCES}

Friesenegger A (1996). Numerische Dichte und Clusterung von Zellen des Gelenkknorpels (Caput femoris und Facies articularis navicularis des Caput tali) in Abhängingkeit von Schichttiefe und Lebensalter. Leipzig: Univ Med Fak Diss, 101.

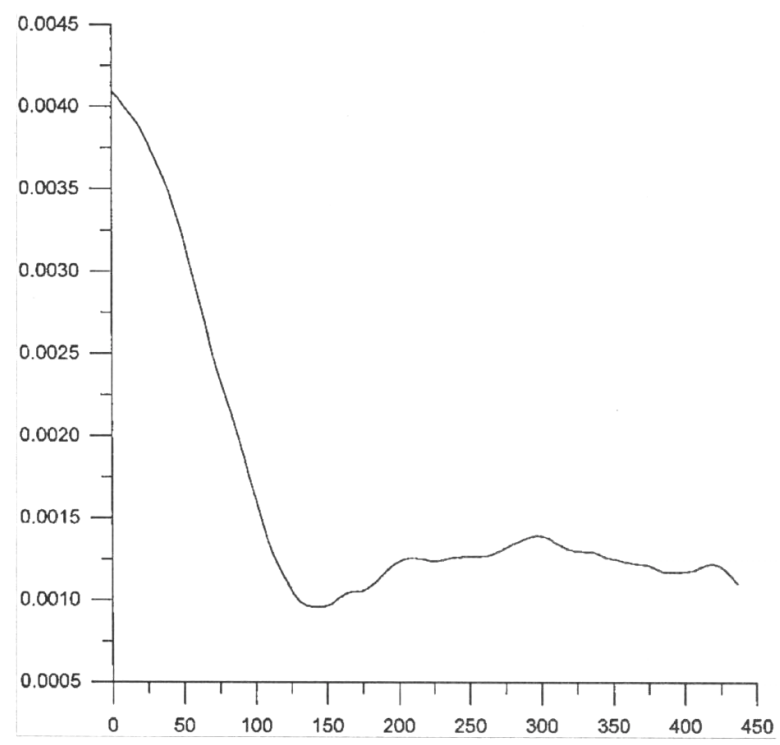

Fig. 6a. The estimated intensity function for the sample given in Fig. 2. for $e_{\lambda}=60 \mu \mathrm{m}$.

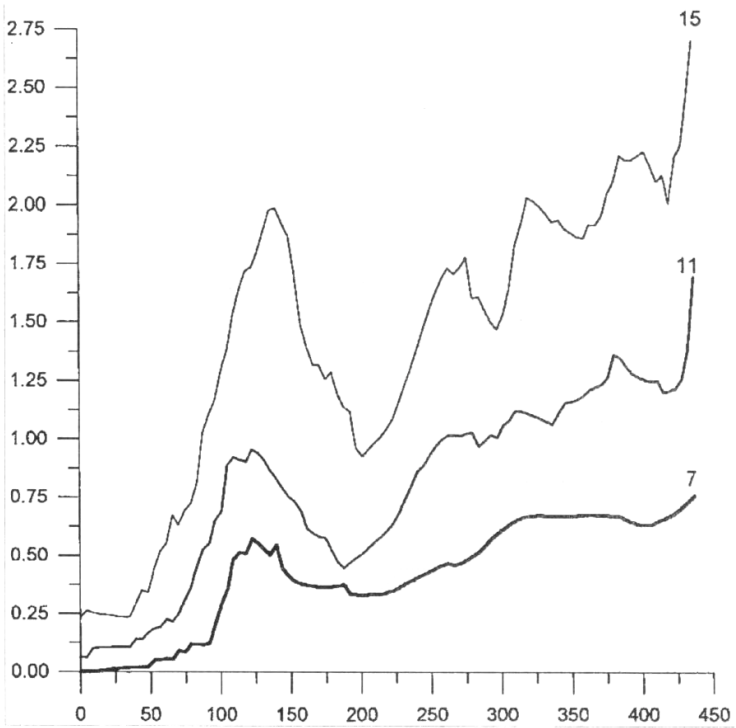

Fig. 6c. The cluster density for the sample given in Fig. 2. for variable cluster radii with else equivalent data as in $b$.

Hahn U, Micheletti A, Pohlink R, Stoyan D, Wendrock H (1999). Stereological analysis and modelling of gradient structures. J Microsc 195(2):113-24.

Löffler S, Ranke TP, Rother P (1993). Alternsveränderungen systematischer Inhomogenitäten des Gelenkknorpels. Leipzig: Z. für Gerontologie 26:247-50.

Löffler S, Rother P, Burkhardt M, Herrmann H (1995). Aging changes of main orientation and anisotropy of articular cartilage cells. Vienna: Aging Series 5:347-52. 
Ranke I, Ranke TP. Quantitative Erfassung eines Differenzierungs prozesses im Knorpelgewebe - am Beispiel von Gießbeckenknorpel des Larynx und Spornknorpel der Carina tracheae. Leipzig Univ: Med Fak Diss, 86.

Schreitter SP (1993). Quantitative Charakterisierung der systematischen Inhomogenität des hyalinen Knorpels (Anlage der Fußwurzel, Gelenkknorpel) an Hand der numerischen Dichte der Chondrozyten sowie ihrer räumlichen Verteilung. Leipzig Univ: Med Fak Diss, 70.

Silverman BW (1986). Density Estimation for Statistics and Data Analysis. London, New York: Chapman \& Hall, 1986.

Stoyan D, Kendall WS, Mecke J (1995). Stochastic Geometry and its Applications. J Wiley \& Sons. 